\title{
Social, health, education and economic effects of COVID-19 on adolescent girls in Kenya: Results from adolescent surveys in Kilifi, Nairobi, Wajir, and Kisumu Counties, October 2020
}

Population Council

Follow this and additional works at: https://knowledgecommons.popcouncil.org/departments_sbsr-pgy How does access to this work benefit you? Let us know!

\section{Recommended Citation}

"Social, health, education and economic effects of COVID -19 on adolescent girls in Kenya: Results from adolescent surveys in Kilifi, Nairobi, Wajir, and Kisumu Counties, October 2020," COVID-19 Research \& Evaluations brief. Nairobi: Population Council, 2020. 


\title{
Social, health, education and economic effects of COVID -19 on adolescent GIRLS in Kenya
}

\author{
Results from adolescent surveys in Kilifi, Nairobi, Wajir, and Kisumu \\ Counties October 2020
}

\section{Highlights}

Adolescent Knowledge of COVID-19 risk factors, symptoms and preventive behaviors was mixed. Over half of respondents knew you can become infected and not show symptoms, lowest in Wajir (40\%), as well as that people without symptoms can be contagious, lowest in Kilifi (34\%).

Adoption of preventive behaviors most adolescents left home at least once in the past week, highest in Nairobi (86\%) and lowest in Wajir (48\%). However, most are wearing a mask (over $95 \%$, except in Wajir at 46\%), and staying 1-2 meters apart (over 75\%, except in Wajir at 38\%).

School work Almost all (85\%) adolescents were in school before COVID-19 related closures, lowest in Wajir (78\%). Almost all (93\%) said they would definitely return when schools re-open, and most were doing some learning from home (lowest in Wajir at 50\%). However, risk factors for dropping out are present: overall $11 \%$ are working, in Wajir $65 \%$ of those not doing school work said the school had not provided lessons, while in Kilifi $37 \%$ of those not doing school work said they were needed for chores. Many were spending more time on household chores such as cooking, cleaning, collecting water or firewood, or washing clothes, particularly older adolescent girls.

Food insecurity is a threat: over half skipped meals in the week prior to the survey or are eating less due to COVID-19, and 59\% report they were receiving a meal at school prior to school closures. Older adolescent girls were most likely to report receiving this meal.

Health needs: in Nairobi, Kisumu and Kilifi $11 \%$ are forgoing necessary health services, higher among female than male adolescents. Almost all adolescents report feeling threatened or anxious when they think about Coronavirus, and around half report feeling down or depressed in the last 2 weeks.

\section{Introduction}

Adolescents (10-19 year olds) comprise about 24 percent of Kenya's population. Marginalized adolescent girls in particular, face considerable risks and vulnerabilities that affect their educational status, health and general well-being. With the continued spread of COVID-19 in Kenya, women and girls face increased and multiple challenges including physical and psychological violence related to family confinement, isolation and economic vulnerability.

The Government of Kenya through the Presidential Policy \& Strategy Unit (PASU), Executive Office of the President, in partnership with Population Council Kenya, has undertaken the study that aims to document the social, health, economic and education effects of COVID-19 mitigation measures on adolescents in Kenya, with a particular focus on girls. 


\section{Study Cohorts and Sites in Kenya}

Between June and August 2020 a COVID-19 mobile-phone survey was completed in four locations across Kenya with adolescents ages 10-19 years ( $n=3,921)$. A total of 1,022 interviews were completed in Nairobi, 602 in Kisumu, 1,063 in Kilifi, and 1,234 in Wajir (see Map). The average age was 16 years and $72 \%$ were female. Households in each site were sampled from underlying cohort studies conducted by the Council. The map highlights cumulative COVID cases as of July 2020, and highlights the four study locations. These were

1 Nairobi: five informal settlements were sampled across Nairobi from the Adolescent Girls Initiative-Kenya (AGI-K) and the NISITU study.

Kisumu: is a city located along lake Victoria. Households were part of the DREAMS program and located in an informal settlement and peri-urban area.

Kilifi: is a largely rural county located on the coast of Kenya, north of the city of Mombasa. Households were part of the Evaluation of the Nia Project cohort.

Wajir: households across 79 villages in drought prone, rural, northern Wajir county were sampled from the AGI-K study cohort.

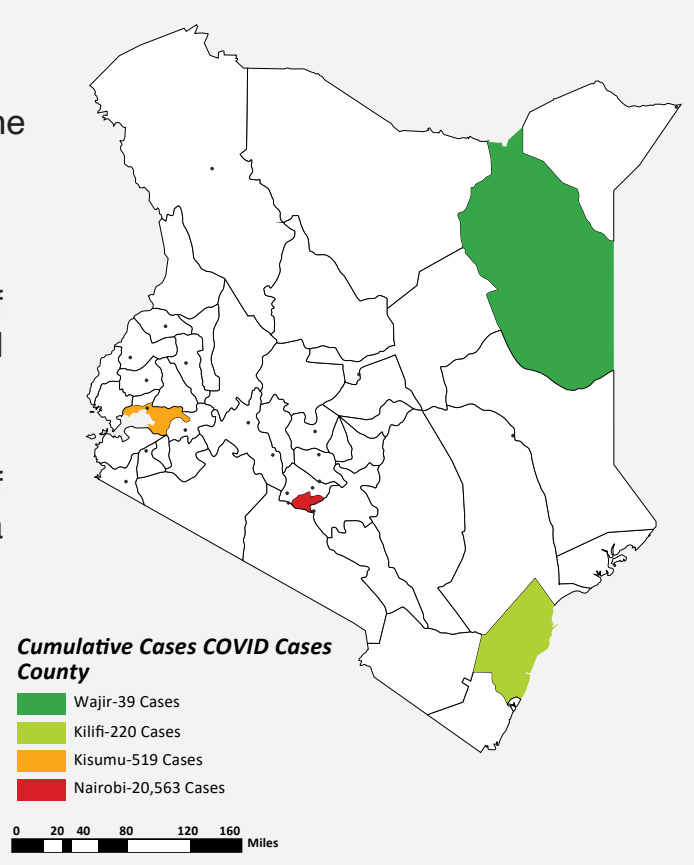

\section{Knowledge, Attitudes, and Perceived Risk of Infection}

Six months into the pandemic, most adolescents across the four sites perceived their risk of getting COVID-19 to be low, and basic knowledge of the disease was ubiquitous. However, the specifics were mixed; for example, while $94 \%$ could name one key symptom (fever, cough, or difficulty breathing) only $23 \%$ named all three (only $6 \%$ in Wajir). Adolescents in Wajir also had lower knowledge of prevention behaviors. While knowledge and adoption of preventive behaviors is highest in Nairobi and Kisumu, the risk is also highest in these urban/peri-urban areas that experience local transmission of COVID-19. While knowledge and adoption are lowest in Wajir, this region is also extremely rural and has had very few cases.

- Perceived risk was low; about a third (31\%) of respondents in Nairobi said they are high risk, $18 \%$ in Kisumu, $12 \%$ in Kilifi, and only $3 \%$ in Wajir County. Participants who said they are low risk mainly cite this is because they are following government guidelines (washing hands, wearing masks), and those who say high risk cite that they interact with a lot of people or live in a crowded place, reflecting an accurate understanding of risky behaviors

- Knowledge of preventive behaviors overall was high, most respondents knew to wash hands with soap, followed by wear a mask. Less than a third reported that staying home unless urgent was recommended. Compared to males, female respondents had higher knowledge of these preventive behaviors.

- Knowledge of asymptomatic transmission was mixed, with about half not knowing you can be infected with Coronavirus and not show symptoms; highest in Nairobi (62\%) lowest in Wajir (40\%). Overall, many respondents did not know "only people with symptoms are contagious" was false, lowest in Kilifi (66\%) (over 50\% in the other sites).

Ensuring that adolescents have access to accurate information is critical, tailored to the risks and realities of the communities they live in. As schools begin a phased reopening in October 2020, ensuring preventive behaviors are promoted to adolescents will be important to stop transmission at school but also within the homes of adolescents. Most adolescents perceive their risk of getting COVID-19 to be low. Previous behavioral research has shown that if perceived risk is low, individuals are less likely to adopt a preventive behavior. Adolescents are taking public transportation, many live in intergenerational households, and many say they will return to school. Ensuring they understand how to use public transportation safely, when and where to wear masks in school and in public, and the possibility of asymptomatic transmission is critical to ensure they adopt healthy behaviors protecting themselves and their households from potential COVID-19 transmission. 


\section{Knowledge, Attitudes, and Perceived Risk of Infection}

Most adolescents (68\%) report leaving home at least once in the week prior to the survey, but most reported that when they did, they wore a mask and/or stayed at least 1-2 meters apart. While findings suggest knowledge of preventive behaviors did translate to adoption of these behaviors, overall, many adolescents still report leaving their home with some taking public transportation and interacting with many people.

It is likely impossible for most Kenyans to stay home completely, as many lack electricity or refrigeration, and leave home daily to fetch water, food, and may share toilets/latrines with other households. Over a third (35\%) of respondents in Kisumu say they interacted with more than 10 people last time they went out, 26\% in Nairobi and Kilifi (Question not included in Wajir). Older adolescents, and males in particular, had more interactions - this is supported by our published analysis of social contacts in adults in Nairobi that found men had more interactions.

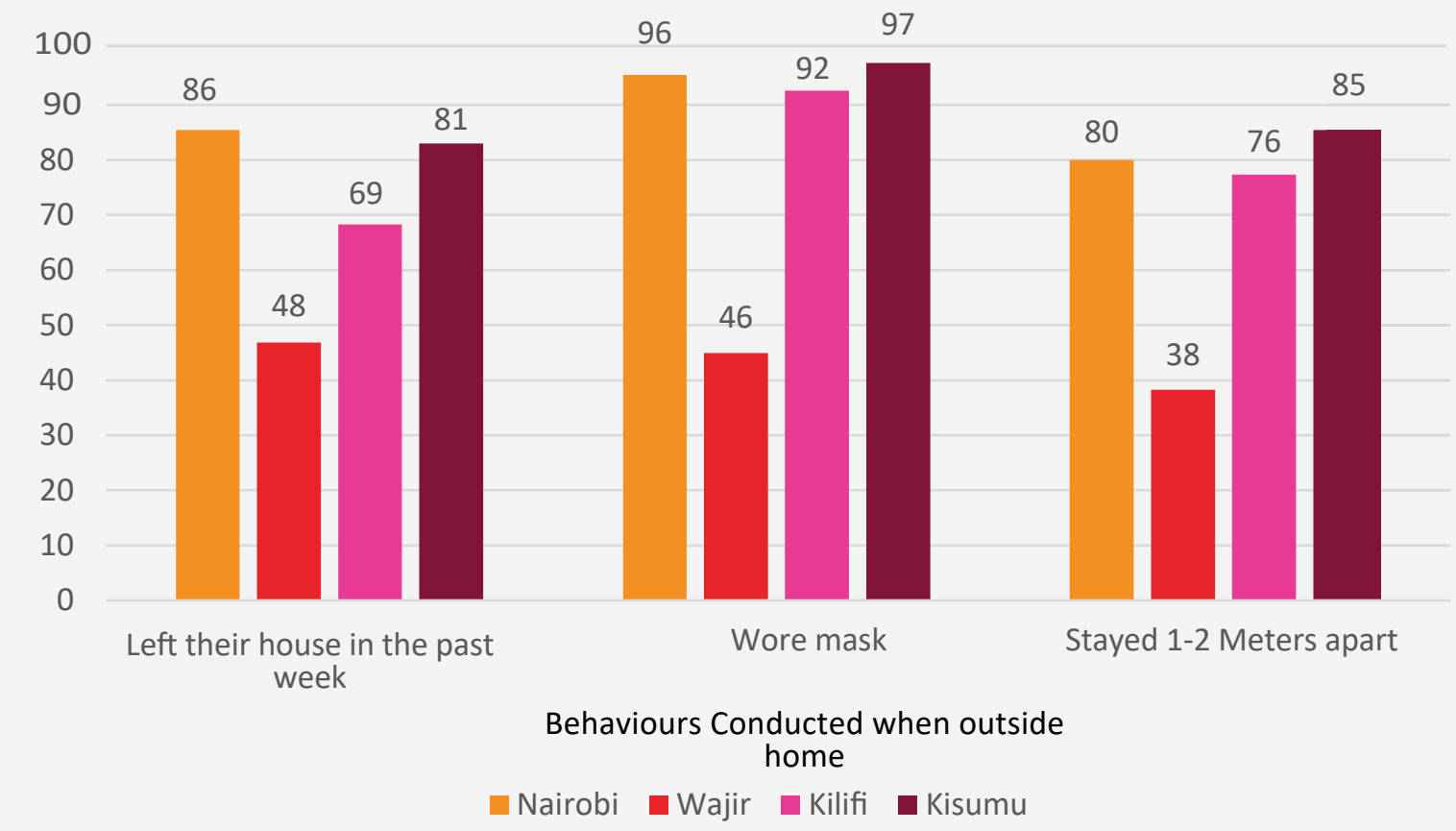

If going out, it is critical to take precautions including wearing face masks, washing hands with soap or using hand sanitizer, staying 1-2 meters apart, and practicing physical distancing. With these precautions in place, it is possible that transmission can remain low, especially if many social interactions are occurring outdoors (e.g., open air markets). However, as businesses and schools reopen it will be critical to highlight the need for physical distancing, because even masks and handwashing may not be effective if people are having many indoor interactions especially in locations with poor ventilation.

Identification of specific tasks assigned to adolescents could help tailor messaging for preventive actions they can take. For example, $19 \%$ of adolescents said they rode public transportation when they went out, this was highest in Kisumu (32\%), Kilifi (20\%), Nairobi (17\%) and $9 \%$ in Wajir. Within each site, older adolescents (15-19 years) were more likely to report riding public transport, with no variation by gender except in Kisumu where females 15-19 years of age were most likely to ride public transportation ( $41 \%$ vs $24 \%$ of males $15-19$ years old). This type of local mobility is allowed in Kenya, but without precautions may lead to increased spread of COVID-19 or implementation of a more severe lockdown policy

\section{Impact of the COVID-19 Mitigation Response}

While most adolescents have not had COVID-19 or know anyone who has, the mitigation policies including physical distancing and lockdown measures have had a considerable economic, health, educational, and social impact on their lives. Schools have been closed since March 2020 and a phased re-opening began in October 2020, but for only three of the twelve grade levels. This means that many adolescents will have missed almost a full school year. Although adolescents report they are confident they will return, it is ultimately not their decision. Several red flags emerged in our survey suggesting that some adolescents may be at risk of dropping out or experiencing challenges related to food security, health and/or safety. 


\section{Education and risk of drop out}

Almost all (85\%) adolescents were in school before COVID-19 related closures, lowest in Wajir (78\%). When asked if they think they will return after school reopens, $93 \%$ across all sites said absolutely. The main reason for not returning (in Nairobi, Kisumu and Kilifi) was not being able to afford school fees (70\%).

Self-reported home-based learning was high, with 4 out of $5(80 \%)$ adolescents reporting they had been doing some learning or schoolwork from home, through different sources. However, the quality and frequency of this remote learning are unclear. Several red flags emerged highlighting high-risk adolescents that may not re-enroll in school when it re-opens without additional support. These include:

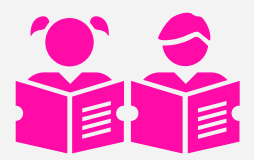

Access to schoolwork: Although most were doing their schoolwork remotely, about $10 \%$ were not doing any, highest in Wajir (50\%). In Wajir, $65 \%$ of adolescents not doing any work said it was because the school had not provided them with any. Across the sites, 'reading other books/schoolwork' was one of the most common responses to where respondents were doing schoolwork, followed by given papers, books or materials by school. This may be a barrier if students must identify these other sources of learning themselves.

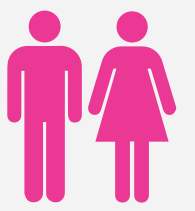

Digital gender divide: overall less than a third of students used a mobile phone for remote learning, but within this group there was a difference by gender (except in Nairobi). Mobile phone ownership was almost exclusively among 15-19 year olds, and was higher for males than females (33\% vs $29 \%)$.

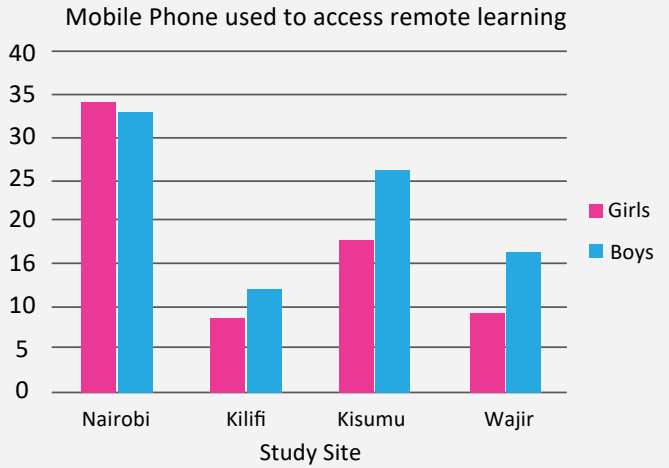

Time use: in Wajir, of the students not able to do any schoolwork $25 \%$ said it was because they were needed to do chores, this was $37 \%$ in Kilifi. Across the sites, respondents report they are spending more time on chores including cooking, cleaning, collecting water or firewood, or washing clothes. Overall, females spent more time on chores than males, although there was some variation (e.g., in Nairobi, younger males spent the most time). Older adolescent girls reportedly spent 3.6 hours/day on average on chores. By site, participants in Wajir spent the most time on chores and in Nairobi the least, although this may have been true pre-COVID-19.

Formal work and income generating activities: Overall, $11 \%$ were involved in formal work and income generating activities since COVID-19 started. This was highest in Kilifi and was highest for adolescent males (18\%). Already working and pursuing income generating activities is of concern since these adolescents may choose to just continue earning money and not return. Their parents, facing
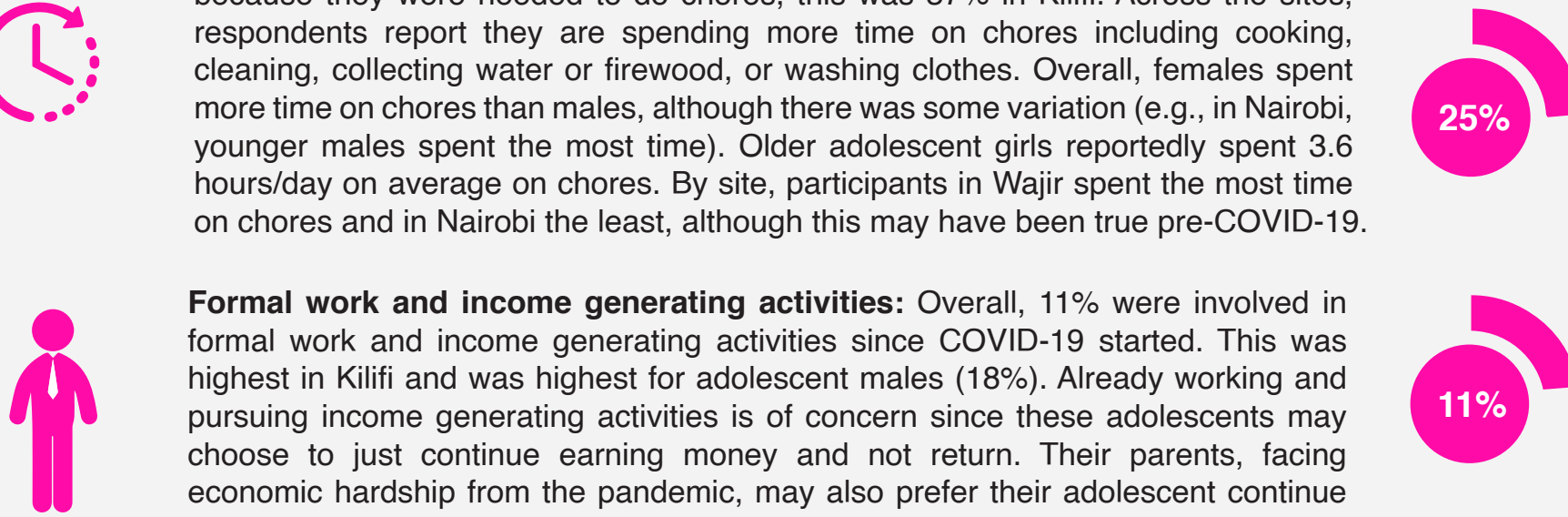
economic hardship from the pandemic, may also prefer their adolescent continue earning money.

These findings highlight challenges in pursuing home-based learning, and the additional risks of formal work and income generating activities that may not allow adolescents to return to schools as they re-open. Potentially once adolescents are able to contribute to household finances, their parents may not want them to return to school. Realistically, many will have fallen behind even if some remote learning has occurred. Waiving or reducing school fees and facilitating a return to school will be critical for adolescents to maintain their trajectories and educational goals 


\section{Food Security, Health and Safety}

Adolescents face additional barriers to their education and well-being, including food security, mental health, and healthcare seeking behavior. For adolescent females, access to menstrual hygiene products is a challenge. And a small proportion face household tension and violence.

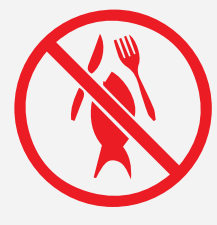

Food Security: In each site, over half of adolescent's report skipping meals or eating less due to COVID-19. This was over three-quarters in Nairobi (78\%), Kisumu $(79 \%)$, and Kilifi $(76 \%)$, and over half in Wajir (55\%). In the week prior to the survey, $45 \%$ said they are skipping meals a couple times per week, and $76 \%$ say this is more often than before COVID-19. Before schools closed, 59\% said they were receiving at least one meal at school. This was highest in Nairobi $(74 \%)$, Kisumu $(68 \%)$, Kilifi $(58 \%)$ then Wajir $(43 \%)$. This was higher for female respondents and older (15-19 year old) respondents.

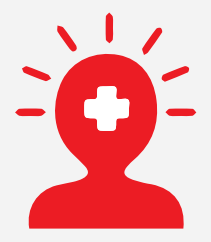

Mental health: There are mental health effects being felt already. Respondents report feeling anxious, threatened, or concerned when they think about COVID-19, and experiencing depressive symptoms in the two weeks before the survey took place. Most say this is more than before COVID-19

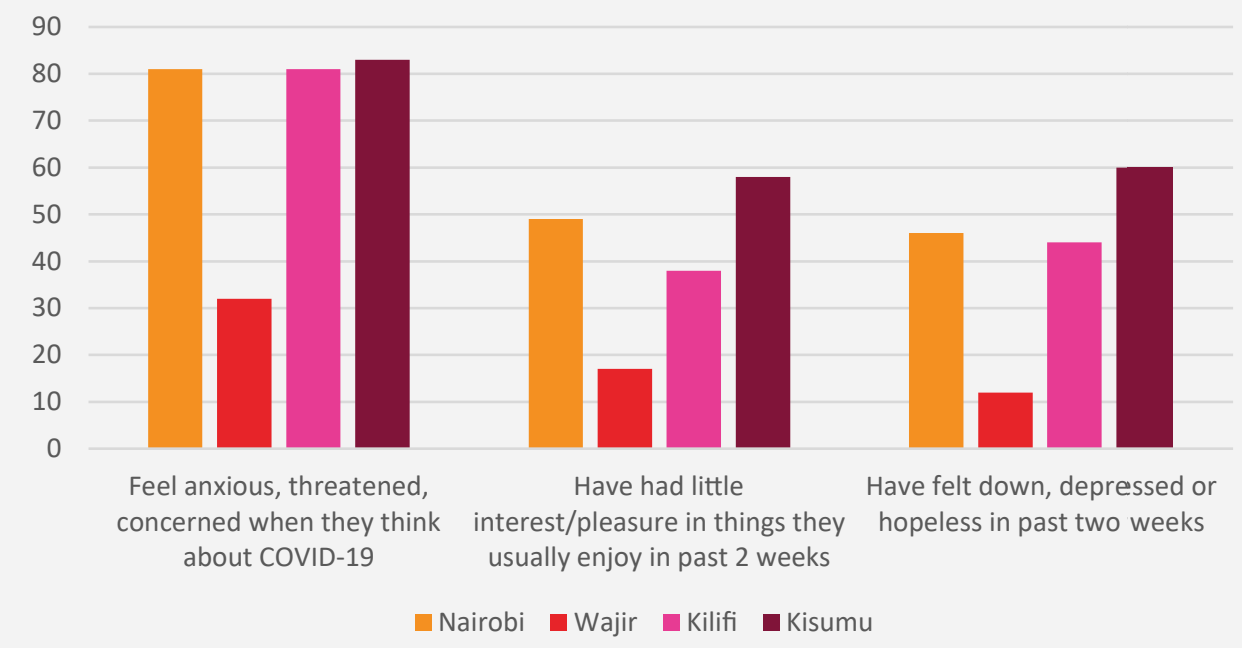

Menstrual Hygiene: Overall, disposable sanitary pads were the most used menstrual hygiene product used (85\%). However, over half of girls in Kilifi $(57 \%)$, Kisumu (54\%), Nairobi (51\%) and over a third in Wajir (34\%) have had challenges accessing their product.

Healthcare seeking: Overall $11 \%$ of adolescents reported forgoing needed health services in the past month, higher for female $(12 \%)$ than male $(9 \%)$ of respondents (excluding Wajir).

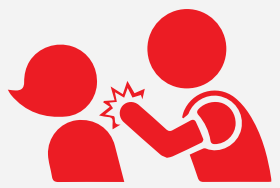

Household tension and violence: $11-24 \%$ report increased tension in the household, $7-12 \%$ report experiencing emotional abuse. For all reports of violence, more than half say it is more than pre-COVID-19. These reports were highest in Nairobi and Kisumu.

- In Nairobi, Kisumu and Kilifi, respondents were asked more detail about their experience of violence. In Kisumu, $12 \%$ of respondents reported experience of emotional violence and $8 \%$ physical violence; in Nairobi, this was $7 \%$ and $5 \%$, respectively; in Kilifi this was $9 \%$ and $4 \%$, respectively. The report of sexual violence was $2 \%$ in all three sites. For all reports of violence, half to three-quarters say it is happening more than pre-COVID-19. 
Food security and mental health are concerns affecting many adolescents across the four sites. With schools closed, many are not getting access to supplemental meals, and this insecurity is compounded by the high economic losses reported that result in many adolescents forgoing meals. A smaller proportion are forgoing health services or experiencing household tension and violence, but these are very high-risk indicators that require urgent attention and support. Facilitating access to health services for adolescents and ensuring access to social protection are necessary but challenging with COVID-19.

While there has not been an increase in teenage pregnancy in these early months of the pandemic, the factors that put girls at risk - being out of school, economic stress in the household and lack of use of family planning - are all there, increasing the importance of such interventions so as to avert longer term increases in teenage pregnancy. To date, Kenya is not implementing any social protection or other services for adolescents.

\section{Conclusions and Recommendations}

Overall, awareness of COVID-19 and adoption of preventive behaviors is high. Most adolescents have not had COVID-19 or know anyone who has, but all are being impacted by the mitigation policies (e.g., physical distancing measures and school closures). With phased school reopening having begun in October 2020, it will be critical to facilitate a return to the classroom, and to address widespread challenges including food insecurity and mental health. Identifying and supporting high-risk youth, particularly those that are working or experiencing household violence, will be critical representing about $10 \%$ of the adolescents interviewed. Recommendations include:

Expand the options for students to access educational materials during the period of school closures so that they can maintain their skills and interest in learning. As schools open, pay close attention to girls at risk of drop-out and older adolescents that have start working during COVID-19 to ensure that they re-enroll in school and complete secondary school. Facilitate and support this return, potentially waiving or reducing school fees.

Distribute cash, food or meals as three quarters of adolescents are missing meals, especially since they are also not receiving meals from school during closures. Cash may also help to alleviate additional impacts of the economic stress in their households.

Many adolescents feel anxious about COVID-19 and are seeing friends less than before, with some experiencing depressive symptoms. Ensuring they have accurate information and support is critical to improve mental health and prevent social isolation

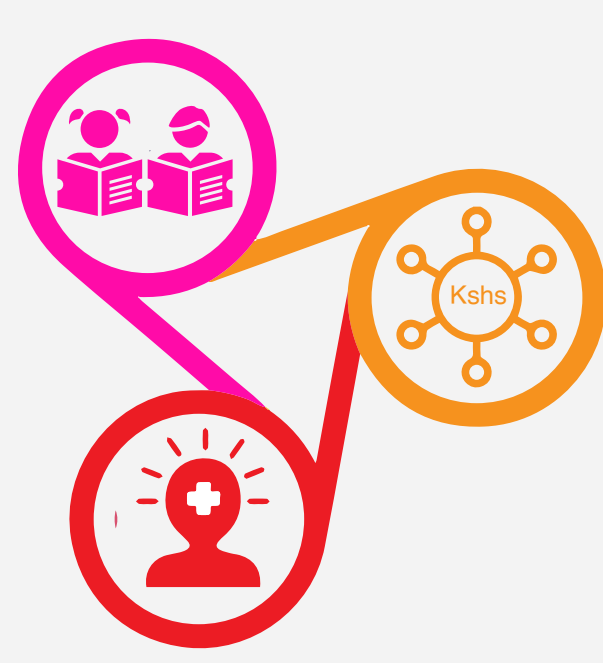

\section{Next Steps}

After completing the first round of quantitative data in each site, the study team held validation meetings with a group of adolescents from each site, as well as the study's Technical Advisory Committee. Qualitative data will be collected from girls, boys, parents and other key stakeholders in each of the four sites, as well as three additional counties to ensure representation from a range of Kenya's regions in November 2020. A second round of quantitative data will be collected in February 2021 to assess the longer-term impacts on school re-enrollment, pregnancy and child marriage. This information will be used to make recommendations to guide adolescent programs and policies in Kenya in the COVID-19 recovery period. 